\title{
ORGANIZACIÓN DE LA SEGURIDAD EN EL PROCESO CONSTRUCTIVO
}

\author{
(ORGANIZATION OF SAFETY IN THE CONSTRUCTION PROCESS)
}

\author{
D. César Mínguez Fernández, Aparejador y Arquitecto Técnico \\ Comsión de Seguridad de SEOPAN \\ ESPAÑA
}

Fecha de recepción: 14-I-93

\section{RESUMEN}

Profundo y complejo estudio sobre la implantación de los procesos de prevención y salud laboral, en el proceso de producción, en la Empresa Industrial de Construcción.

\section{SUMMARY}

Thorough and complex study on the implementation of prevention processes and labour safety in the production process in Industrial Construction Companies
La ejecución de un proyecto de construcción, sea éste de la magnitud y características más exigentes, requiere un estudio "a priori" de todas las unidades de obra en toda su extensión, no solamente en las características técnicas, sino también en el plano económico, el cual conlleva una calidad en los materiales de acabado que determinarán en definitiva los distintos niveles de éxito para el autor del proyecto, para quien lo dirige y para la empresa constructora que lo realiza.

\section{SEGURIDAD Y PLANIFICACIÓN}

En la mayoría de los casos el tiempo de ejecución viene fijado por la propiedad y juega un papel de máxima importancia, puesto que si resulta muy justo nos lleva, en algunos casos, a plantear cambios a materiales más rápidos como, por ejemplo, el prefabricado en la ejecución de la estructura, en los cerramientos, en las distribuciones, etc...; son alternativas que una vez que hayan sido estudiadas y planificadas, son presentadas a la Dirección Facultativa para su aprobación y, una vez aceptadas, es preciso adaptar esas nuevas soluciones a los tiempos ya definitivos en la planificación total de la obra.
Esta planificación conlleva un estudio minucioso de la maquinaria, los medios auxiliares y los medios humanos a emplear, factores éstos de la máxima importancia para conseguir el objetivo marcado.

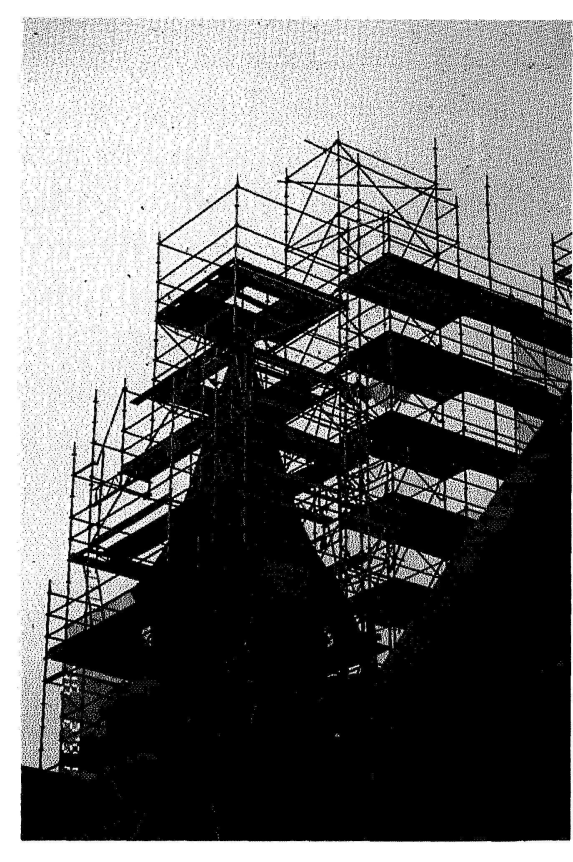

Andamios adecuados a su función. 


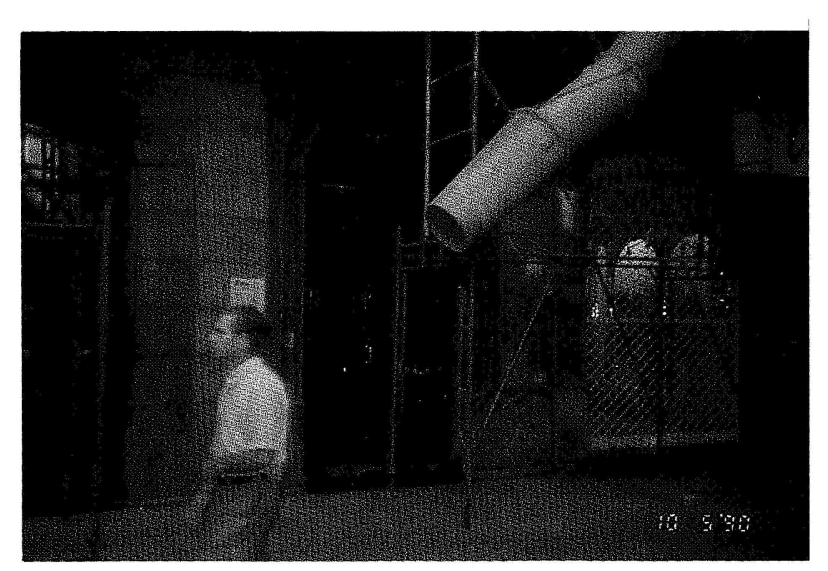

Escombros enviados directamente sobre el peatón.

Se supone que el equipo de especialistas que, con el Jefe de Obra, han elaborado hasta este momento la planificación del proceso constructivo, han previsto en cada fase de trabajo todo lo relacionado con la Seguridad e Higiene, puesto que la Seguridad, o más exactamente la Prevención, ha de formar parte integrante de ese proceso.

En un rápido vistazo a esa planificación, el Prevencionista, el Técnico de Seguridad o el Especialista en Seguridad e Higiene se da cuenta si faltan, por ejemplo: la determinación de los tiempos precisos de grúa para la elevación de las protecciones generales o colectivas; los tiempos precisos de mano de obra para su colocación, mantenimiento y reposición; la mano de obra para la limpieza de las instalaciones de higiene y bienestar; los reconocimientos médicos; la formación; las reuniones del Comité de Seguridad; en fin, una serie de omisiones que realmente pueden desfasar la planificación de la obra. Si así fuera, significaría que el técnico en planificación no ha tenido en cuenta una unidad de obra tan importante, que se llama Seguridad, y que interviene en todo el conjunto del proceso constructivo. A partir de este momento, si realmente queremos hacer una planificación adecuada a las necesidades de la obra, es preciso que iniciemos de nuevo su estudio desde la fase de replanteo y de las instalaciones provisionales de obra para integrar la Seguridad en la Producción, como una unidad más a ejecutar, de forma que todos los elementos que intervienen en el proceso constructivo, bien sean los medios materiales, los mecánicos o los humanos, estén sólidamente unidos con los principios básicos de la Prevención.
Ante los cambios que se produzcan en la metodología de ejecución en cualquier unidad, el planificador consultará con el Técnico de Seguridad para que dé las soluciones óptimas con objeto de llevar a cabo la unidad modificada. Y, a su vez, es preciso definir muy claramente los sistemas, medios o formas de actuación, en lo que a la seguridad se refiere, para que toda persona de la línea de producción la vea como un elemento de ayuda y no como un estorbo para la Producción.

\section{LA SEGURIDAD EN LA LEY}

La entrada en vigor de los Reales Decretos 555/86 y $84 / 90$ ha puesto en marcha este complejo mundo de la Seguridad e Higiene para aquellas empresas que no tenían en cuenta esta materia por no disponer de medios técnicos ni económicos en sus obras. No obstante aún existen algunos sectores que sacrifican la prevención a mayores beneficios económicos; otros se acogen a los vacíos legales del Real Decreto e intentan que los proyectos no alcancen el techo determinado de los 100 millones, para no verse obligados a la realización del correspondiente Estudio y Plan de Seguridad. Otros determinan que el mejor camino a seguir es simplemente limitarse al cumplimiento del papeleo oficial ante los organismos pertinentes, obligando a los constructores a señalar, en los contratos establecidos de obra, que las partidas económicas de la valoración de la seguridad están incluidas en los precios unitarios de los distintos capítulos del Proyecto o, simplemente, alegan que como condición para la firma del contrato de adjudicación es preciso anular los importes económicos a los cuales nos estamos refiriendo.

Todo este bagaje de picaresca, que flota en el sector, no está extendido en todos los casos; no obstante, es preciso que los que creemos en la prevención procuremos por todos los medios que no se extienda con carácter general, evitando así la situación de indefensión en que nos encontrábamos los Aparejadores y Arquitectos Técnicos de Dirección Facultativa, los que actúan en Empresas Constructoras o Inmobiliarias, y los que trabajan en la Administración, antes de la promulgación del mencionado Real Decreto.

Sería fácil decir con los demagogos de la seguridad que, si no se cumplen las normas, se paralice el tajo o la obra completa si es preciso - "no hay problema", es la frase 


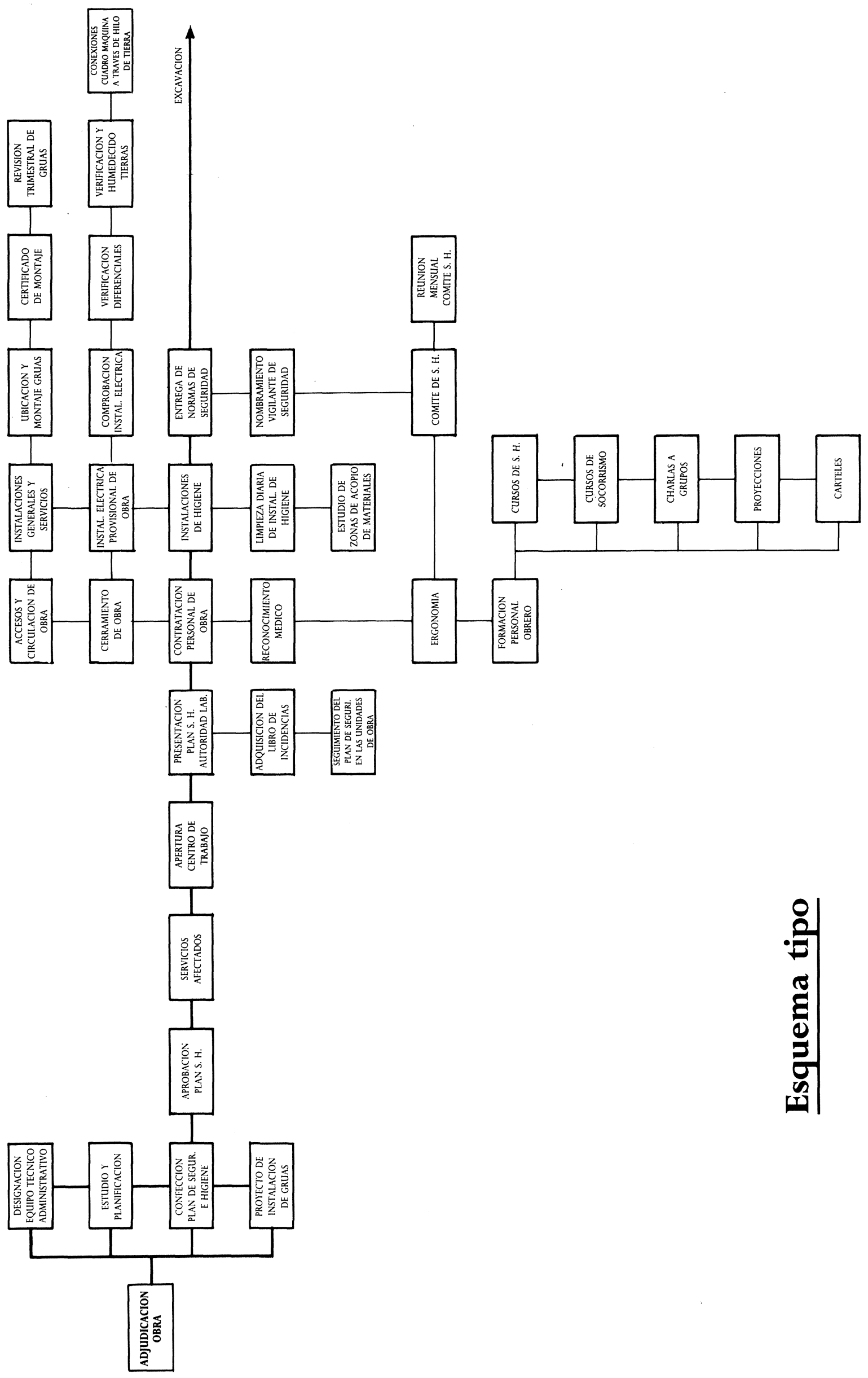


más utilizada-; no obstante, la realidad es otra bien distinta: si no lo indican los Organismos Oficiales, como la Inspección de Trabajo, la obra no se paraliza en la mayoría de los casos.

\section{GENERALIDADES SOBRE UN ESQUEMA TIPO}

De una forma esquemática vamos a tratar de definir una estructura de planificación de la Seguridad, que nos pueda ser útil en todo momento para cualquier tipo de obra; este esquema organizativo, ha de ser apoyado por la planificación del proceso constructivo reflejado en el Plan de Seguridad de la Obra.

El citado esquema está compuesto, en primer lugar, por una descripción inicial que contempla la fase comprendida entre la adjudicación de la obra y la entrega de las

Normas de Seguridad a los componentes de la misma. Esta primera fase la denominaremos en lo sucesivo como preliminar al proceso constructivo y será imprescindible para la ejecución del proyecto.

El resto del esquema organizativo se divide en los ocho grupos siguientes:

\section{Excavación.}

2. Cimentación y saneamiento.

3. Estructura.

4. Cerramiento

5. Cubierta.

6. Distribución interior y acabados.

7? Instalaciones.

8. Remates y finalización de obra.

La unión de todas estas fases, siguiendo paso a paso lo descrito, nos marcará un camino crítico de actuación $\mathrm{y}$ un recordatorio de lo que tenemos que hacer, en todo momento, en el proceso constructivo. Como elemento complementario y de máxima utilidad este proceso ha de llevar implícito no sólo las fechas en las que debemos ejecutar las unidades, sino también aquellas en las que, con la necesaria antelación, los elementos auxiliares, máquinas, etc... deben estar en obra, para evitar así la improvisación en la organización de la seguridad. Estas fechas son fácilmente extrapolables de la planificación general, consiguiendo así la perfecta e indivisa unión del binomio: Producción - Seguridad.

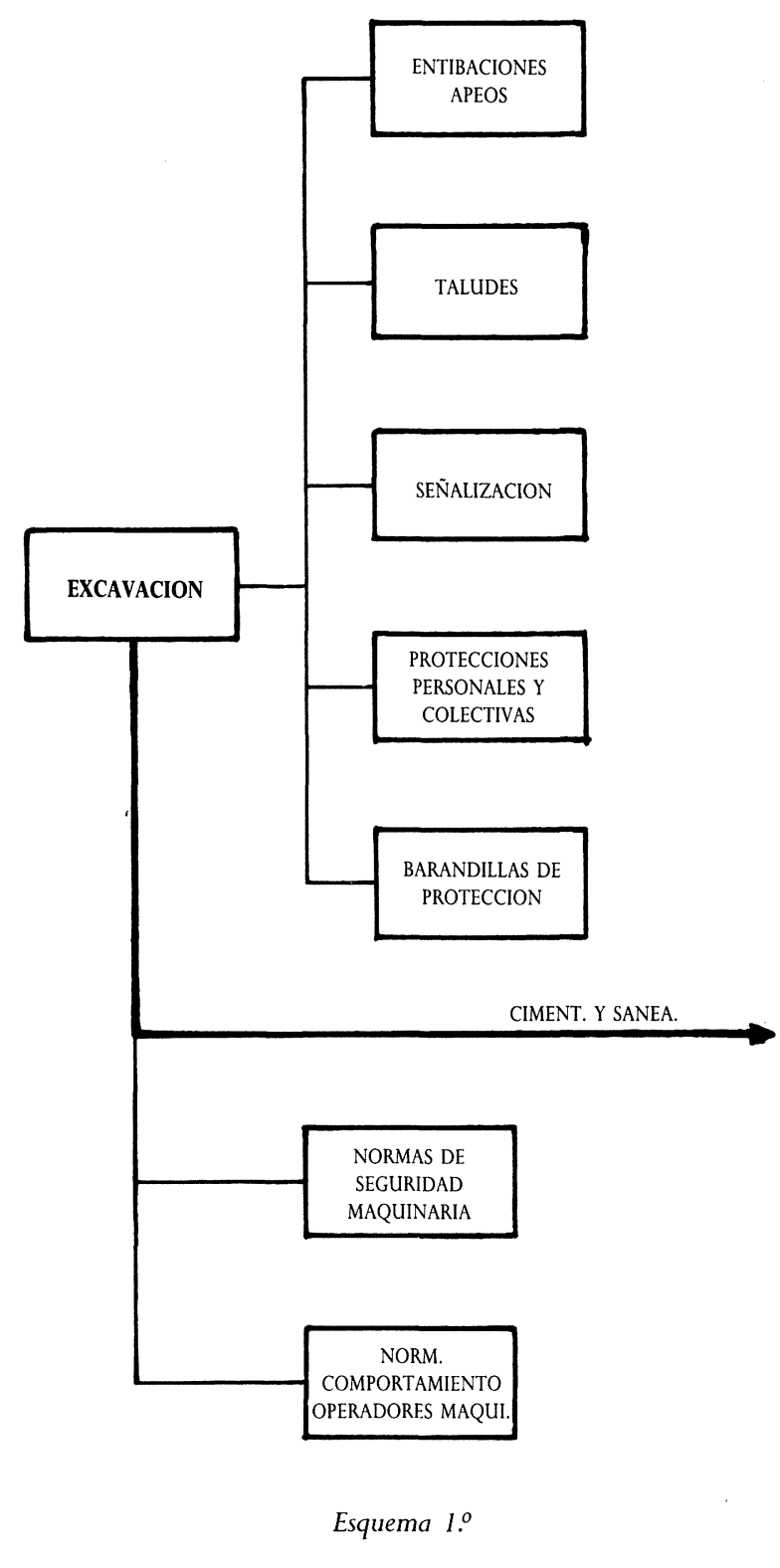

En la oficina donde el Jefe de Obra dispone en sitio bien visible de toda la documentación gráfica, dispondrá también en sitio bien visible de la planificación de la Seguridad, señalando en ella las anotaciones o llamadas oportunas, de la misma forma que lo hace en los gráficos de la planificación general de la obra.

La organización de la seguridad, como cualquier otro tipo organizativo, está formada por una larga cadena de eslabones que han de estar firmemente unidos entre sí. Si por alguna incidencia estos eslabones se ven rotos o detenidos en su propia inercia, todo este sistema que se plantea queda convertido en algo inútil; sería como pretender, por ejemplo, realizar las instalaciones definitivas de un edificio sin haber acabado su estructura. 


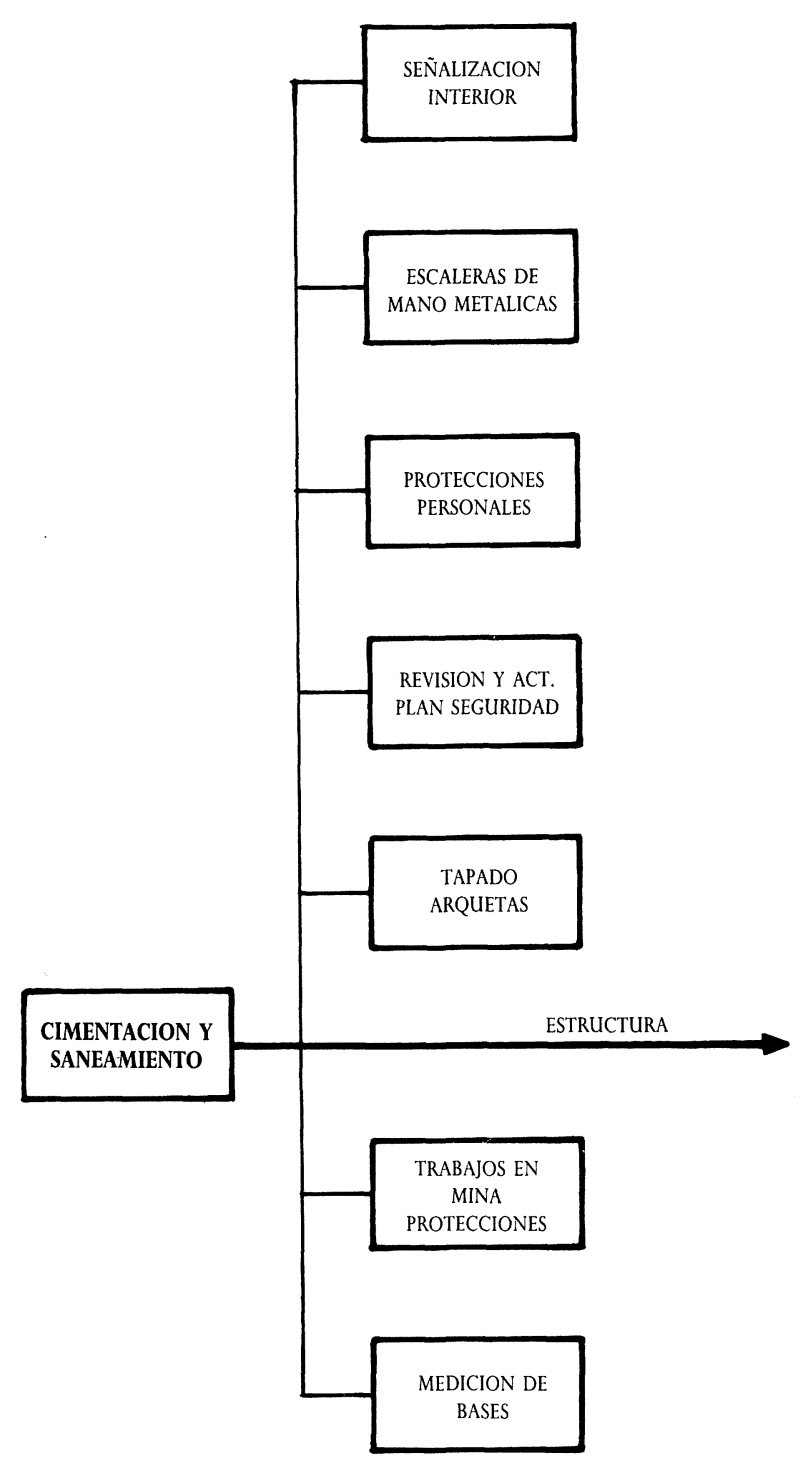

Esquema 2.0

\section{FASE PRELIMINAR}

$\mathrm{Al}$ analizar la fase preliminar del proceso organizativo de la obra hemos de centrarnos en el Estudio de Seguridad, para elaborar el correspondiente Plan de Seguridad, elemento básico, aunque genérico, en la obra a ejecutar, y que nos indicará, en líneas generales, cómo es la obra, qué unidades la componen, el proceso de ejecución estimativo (en la mayoría de los casos no coincide con la realidad) y una extensa documentación gráfica con una ubicación y tipología de grúas que, en general, tampoco coinciden con la realidad, a no ser que el autor del estudio de seguridad sepa de antemano los medios humanos, materiales y mecánicos de que dispone la empresa adjudicataria de la obra. La valoración económica del estudio viene prefijada porcentualmente sobre la ejecución material de la obra, aunque este importe económico, en algunos casos, venga por exceso o por defecto de la valoración real. En otros casos el cálculo porcentual se realiza sobre la Ejecución por Contrata, donde se incluyen los porcentajes de beneficio industrial, los gastos generales e incluso el IVA, incrementando así la valoración de la seguridad en un $30 \%$ como mínimo. Es indudable que si continuamos con este sistema de incrementar los costos, conseguiremos entre todos romper los principios básicos de la Seguridad y, por tanto, desvirtuar el Real Decreto 555/86.

La ejecución del Plan de Seguridad ha de ser realizada respetando las cuantías económicas que se fijan en el Estudio de Seguridad; su estructura ha de ser totalmente coherente con el proceso constructivo, analizando, estudiando y planificando todas las unidades de la obra, y expresando con todo detalle los medios materiales y mecánicos que se van a emplear, las medidas preventivas necesarias que se van a tomar, así como una descripción de la unidad a ejecutar.

Es imprescindible para la apertura de un Centro de Trabajo la presentación, a la Autoridad Laboral, de una copia del Plan de Seguridad aprobado por el autor del Estudio de Seguridad. Sin embargo, aunque la normativa vigente así lo dice, existe un margen de un mes desde que se solicita la referida autorización hasta la presentación de la citada documentación. La nueva Ley de sanciones administrativas contempla una propuesta de sanción de $\mathbf{5 0 0 . 0 0 0}$ pts. para aquellas empresas que no cumplan con este requisito.

El estudio de los Servicios Afectados, que pueden incidir en la construcción de la obra, juega un papel muy importante, ya que es preciso saber dónde y cómo se encuentran las líneas eléctricas, saneamiento, gas, agua, etc... para tener previstos todos los sistemas de desvíos, apuntalamientos, apeos, etc..., evitando sorpresas e improvisaciones. Existen en el mercado unos detectores de campo que son de gran ayuda cuando no se sabe exactamente a qué profundidad y en qué dirección se encuentra.

La normativa vigente determina, en la Orden de 20 de septiembre de 1986, la adquisición y uso del Libro de Incidencias, documento que tiene que ser facilitado por el Colegio Profesional que vise la ejecución del Estudio 


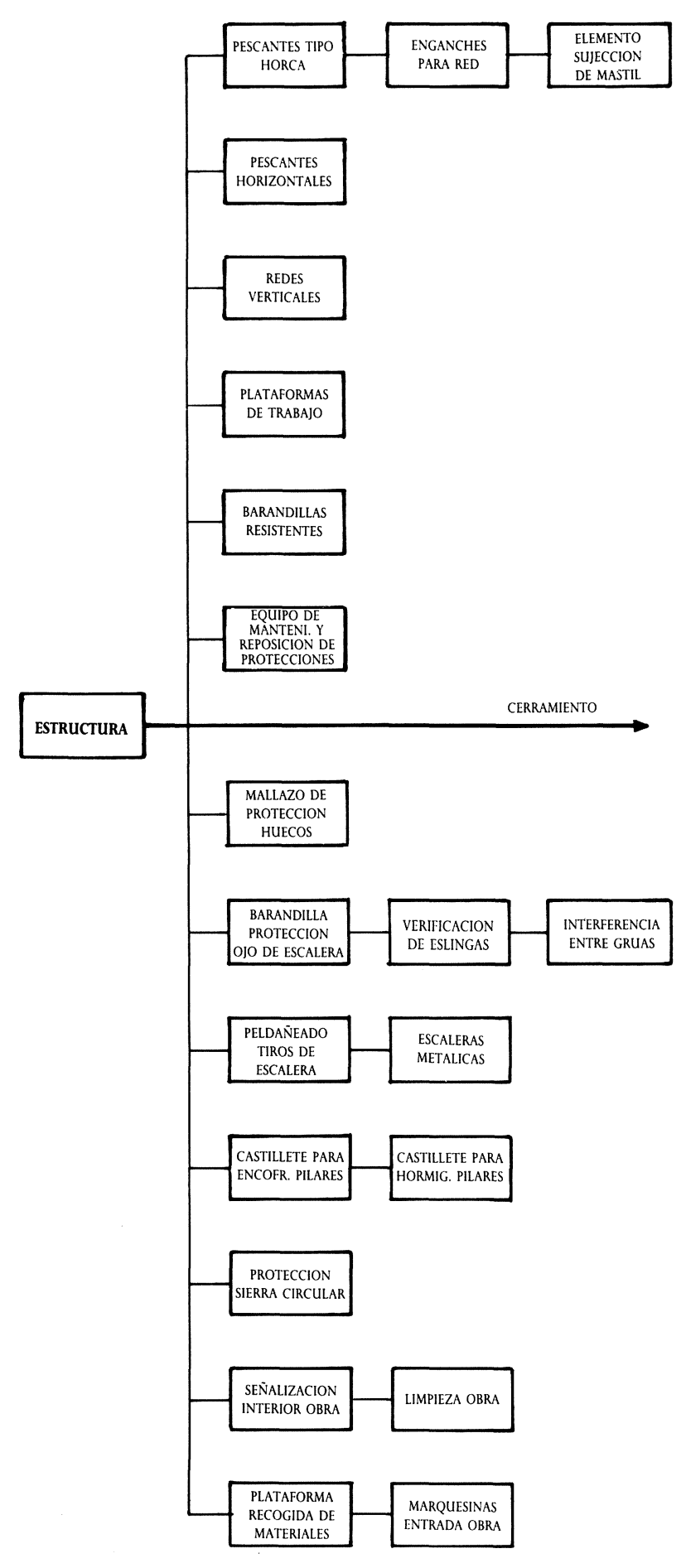

Esquema $3 .^{\circ}$

y dé la posterior aprobación del Plan. Dicho libro ha de permanecer siempre en la obra a disposición de la Dirección Facultativa, Contratista Principal del Comité o Vigilante de Seguridad e Higiene, Subcontratista y Técnico del G.T.P., para que puedan señalar en él las anomalías que existan en la obra y las correcciones que el contratista ha de realizar en todos los aspectos de Seguridad e Higiene. El citado libro dispone de un original para el contratista, y tres copias para: Inspección de Trabajo, Dirección Facultativa y Vigilante o Comité, o representante de los trabajadores.

Antes de la contratación del personal obrero es preciso realizar, según indica la Legislación, los reconocimientos médicos que determinarán el estado físico en que se encuentra el trabajador y poder definir si es apto o no. A partir de este momento, el médico de la empresa con el Técnico de Seguridad realizarán el estudio ergonómico correspondiente para encajar a la persona en el sitio adecuado a su capacidad física y psíquica. Antes de incorporarse a su puesto de trabajo, el trabajador ha de ser informado y puesto al corriente de la labor que va a realizar, y de las Normas de Comportamiento referente a los medios de protección personal y general que debe cumplir en todo momento, obligándose a la utilización de las protecciones personales y al mantenimiento de las protecciones colectivas instaladas para toda la población obrera del centro de trabajo.

$\mathrm{Al}$ iniciar las instalaciones provisionales de obra nos encontramos con la necesidad de llevar a efecto su cerramiento, medida de total y absoluta necesidad para la protección de las personas ajenas a la obra, y obligatoria en todos los Centros de Trabajo. Es preciso a la vez determinar minuciosamente los accesos a la obra, así como las áreas o zonas de acopio y circulación de vehículos, con las correspondientes señalizaciones precisas para estos casos.

La instalación eléctrica provisional de obra ha de ser realizada por un instalador autorizado, que ha de cumplir todos los requisitos que exige la compañía eléctrica suministradora, con base en el Reglamento de Alta y Baja Tensión. Dicha instalación debe ser respaldada por el Dictamen de Industria y proyectada por un Ingeniero Técnico Industrial.

En cumplimiento de la I.T.C. de aparatos elevadores, es necesario elaborar un Proyecto de Instalación antes de la colocación de las grúas necesarias para la obra. Además, es preciso obtener de la casa de montaje un certificado que acredite que la máquina ha sido instalada con todos los requisitos que determina esa Instrucción. Posteriormente, con una periodicidad de seis meses, se ha de revisar totalmente la máquina y obtener el correspondiente certificado de revisión. 


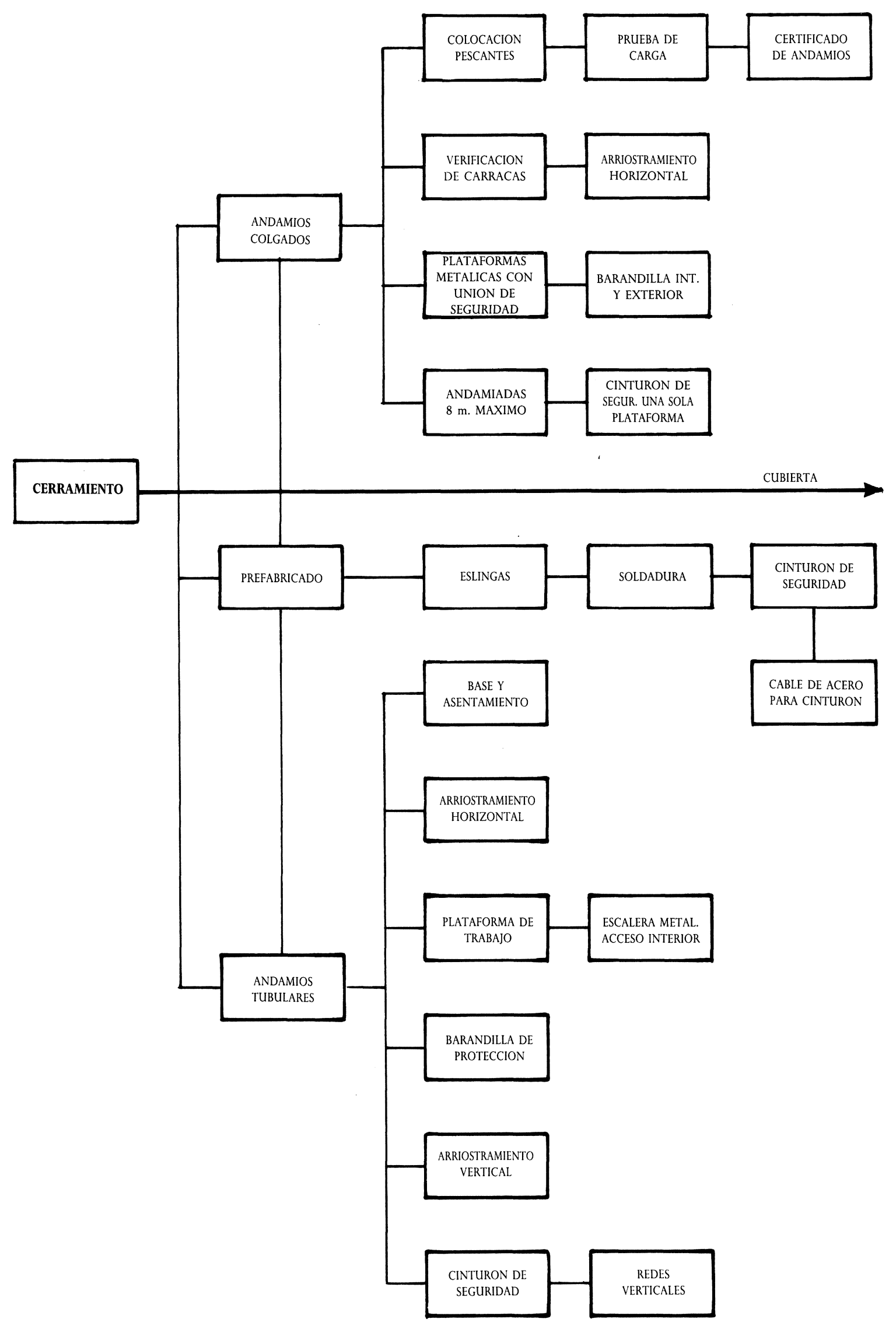

Esquema $4 .^{\circ}$ 
Las instalaciones de higiene y bienestar se llevarán a cabo con arreglo al número de obreros punta que sean precisos para la realización de la obra. Las superficies, dotación y número de aparatos sanitarios, vienen determinados en el Plan de Seguridad según se especifica en la Ordenanza de Seguridad e Higiene en el Trabajo. Dichas instalaciones serán limpiadas diariamente por el personal designado para ello.

Como una acción más a desarrollar, en el Plan Organizativo de la Seguridad, es preciso planificar los tiempos necesarios para la formación de la población obrera a todos los niveles; para ello es preciso a través de toda la obra impartir cursillos o charlas que expongan en primer lugar las reglas mínimas de la Seguridad, incidiendo en aquellas unidades que se están realizando en ese momento con los medios a emplear, y haciendo uso, si es preciso, de lo indicado en el Plan de Seguridad como recordatorio a todos los componentes de la obra, consiguiendo así que este documento esté vivo en todo momento y no se convierta en un trámite más que la Normativa exige.

En esta fase preliminar de la obra, es obligatorio el nombramiento del Vigilante de Seguridad, cargo que es preciso comunicar a la Delegación de Trabajo para su conocimiento. A medida que crece el númerro de obreros, y con arreglo a lo que determine el Convenio Colectivo de la región donde esté ubicada la obra, se nombrará el Comité de Seguridad e Higiene, el cual se reunirá al menos una vez al mes y, con carácter extraordinario, cuando lo convoque su Presidente, motivado por un hecho de máxima importancia en la obra, como es un accidente grave o mortal. Los componentes de este Comité están determinados por el número de personas que indique aquel Convenio Colectivo. Los puntos más significativos que se recomienda analizar son: análisis de los accidentes acaecidos desde la reunión anterior, estudiando los sistemas y protecciones para que estos accidentes no se presenten más; actualización y seguimiento del Plan de Seguridad, proponiendo nuevos cambios o sistemas que se demuestren superiores a los previstos en el Plan; rueda de preguntas y sugerencias de los componentes, con objeto de mejorar el Plan Preventivo y, a la vez, organizar la Seguridad desde arriba hacia abajo y viceversa.

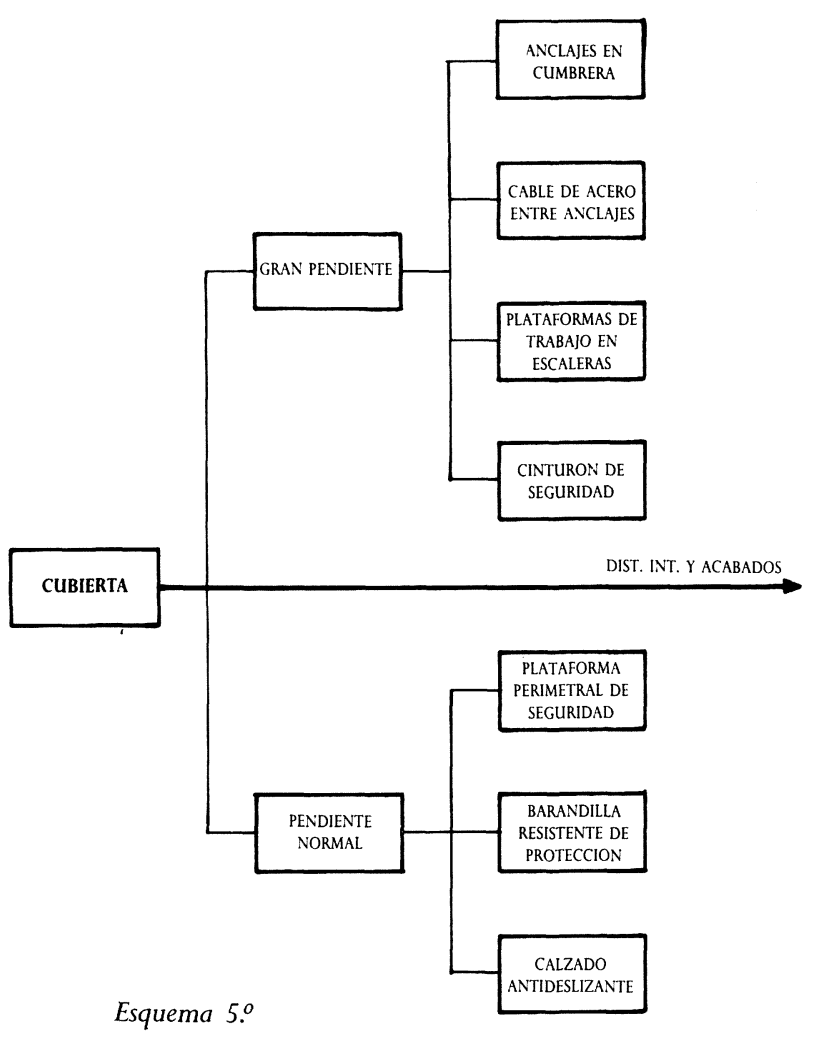

\section{CRONOLOGÍA DE LA SEGURIDAD POR UNIDADES DE OBRA}

Concluida esta fase preliminar al proceso constructivo, vamos a tratar de comentar los esquemas gráficos, desde la fase de Excavación hasta la fase de Remates y Finalización de obra, dentro de este entorno que nos ocupa, haciendo hincapié en que, durante todo este proceso, como factor común, se han de tener muy presentes los tres factores básicos de todo Plan Preventivo: control y seguimiento del Plan de Seguridad, reuniones mensuales del Comité de Seguridad, y Formación del personal obrero.

\section{Excavación}

La unidad de excavación encierra un sin fin de problemas, aun cuando esté definido en el Plan de Seguridad. Siempre nos encontraremos con sorpresas derivadas del estado del terreno, de su cohesión y de los servicios afectados que nos podemos encontrar. Los apeos, entibacio- 


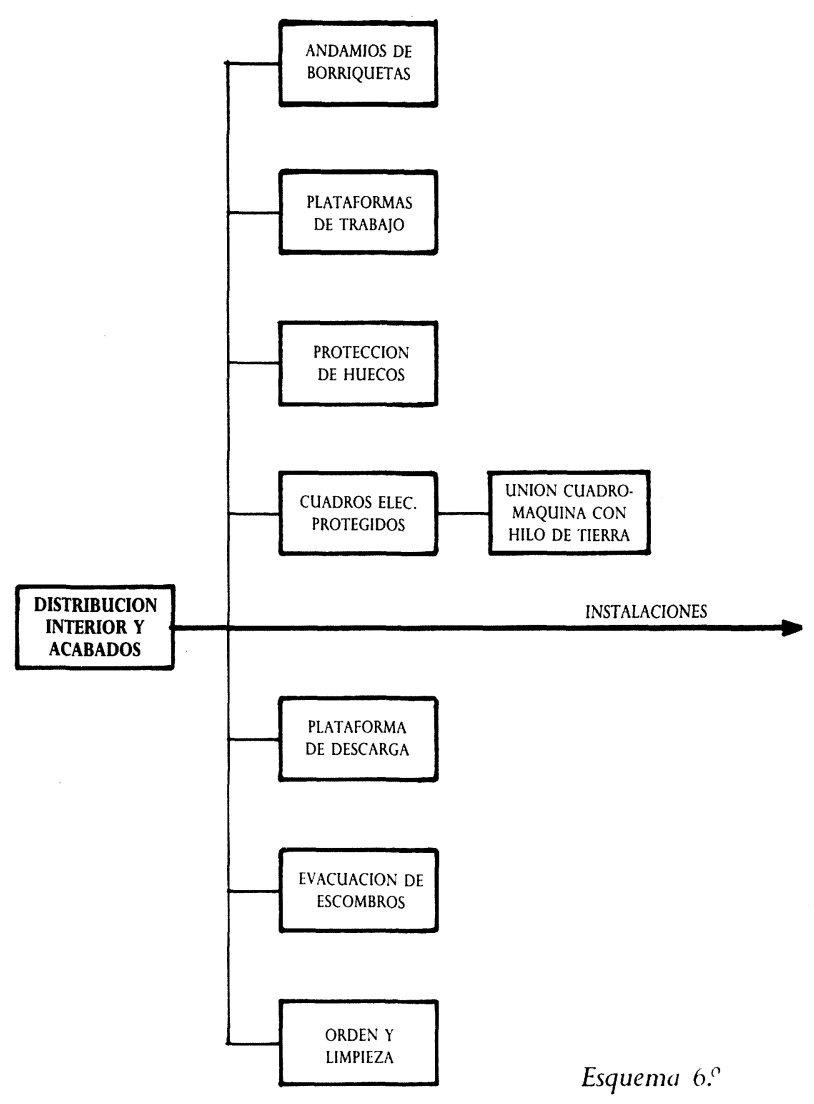

nes, taludes, etc..., son sistemas que tienen que estar perfectamente definidos en el Proyecto General de la Obra y, aunque no es un tema específico de la Seguridad e Higiene, está muy unido a ella; es muy importante tener en cuenta la opinión del Técnico de Seguridad para tomar soluciones, no solamente preventivas, sino de verdadera emergencia. En esta unidad de obra es recomendable proponer un cambio del sistema de ejecución cuando sabemos, por los correspondientes estudios geológicos, que el terreno no responde a lo proyectado. A veces es mucho más rentable cambiar el sistema de ejecución, aunque sea más costoso que el inicialmente previsto, por el alto riesgo que se puede presentar.

\section{Cimentación y saneamiento}

La unidad de cimentación y saneamiento está muy unida a la decisión que se tome en la unidad anterior y, por consiguiente, no es preciso hacer ningún comentario, salvo incidir en lo señalado en los distintos eslabones marcados gráficamente de la cadena Organizativa de la Seguridad.

\section{Estructuras}

Para las estructuras, ya sean de hormigón, metálicas, mixtas y prefabricadas, donde estará presente el factor común mencionado en el apartado anterior, es preciso añadir, además, el equipo de mano de obra para la conservación y mantenimiento de todas las protecciones colectivas instaladas en la correspondiente unidad de obra. En el esquema que define esta actividad, queda reflejado cómo y cuándo debemos tener presentes todos los medios preventivos en esta peligrosa unidad de obra, no siendo preciso mayor comentario a lo expresado en el mencionado gráfico.

Cuando sea necesaria la utilización de más de dos grúas, se plantea el problema de los cruces de las cargas, y será preciso definir una red de preferencia para la entrada de cada grúa en las zonas comunes. Existe en el mercado un sistema computerizado que resuelve este problema, evitando toda interferencia o colisión que se pueda presentar, y anulando así cualquier error humano que pueda originar un accidente múltiple.

\section{$4^{\circ}$ Cerramiento}

La problemática que se plantea en la unidad de cerramiento es también muy amplia, siendo lo más significativo el uso y colocación de andamios, en toda la extensión de la palabra, y donde el cinturón de seguridad ocupa un plano importante. El uso de la eslinga y de los medios de elevación son de máxima importancia, y de ellos depende que se produzcan accidentes que en la mayoría de los casos son mortales. Aunque en el esquema organizativo no se menciona el muro cortina, éste encierra una problemática similar a lo expresado en la unidad mencionada, ya que los elementos auxiliares que intervienen en esa unidad son, de igual forma, los andamios colgados, mecánicos o eléctricos, existiendo además, como variante, la utilización de un medio auxiliar consistente en una plataforma volada, perfectamente sujeta a la estructura a través de unos puntales telescópicos, donde el trabajador efectúa todos los sistemas de fijación de la estructura auxiliar para adosar el cerramiento de vidrio. 


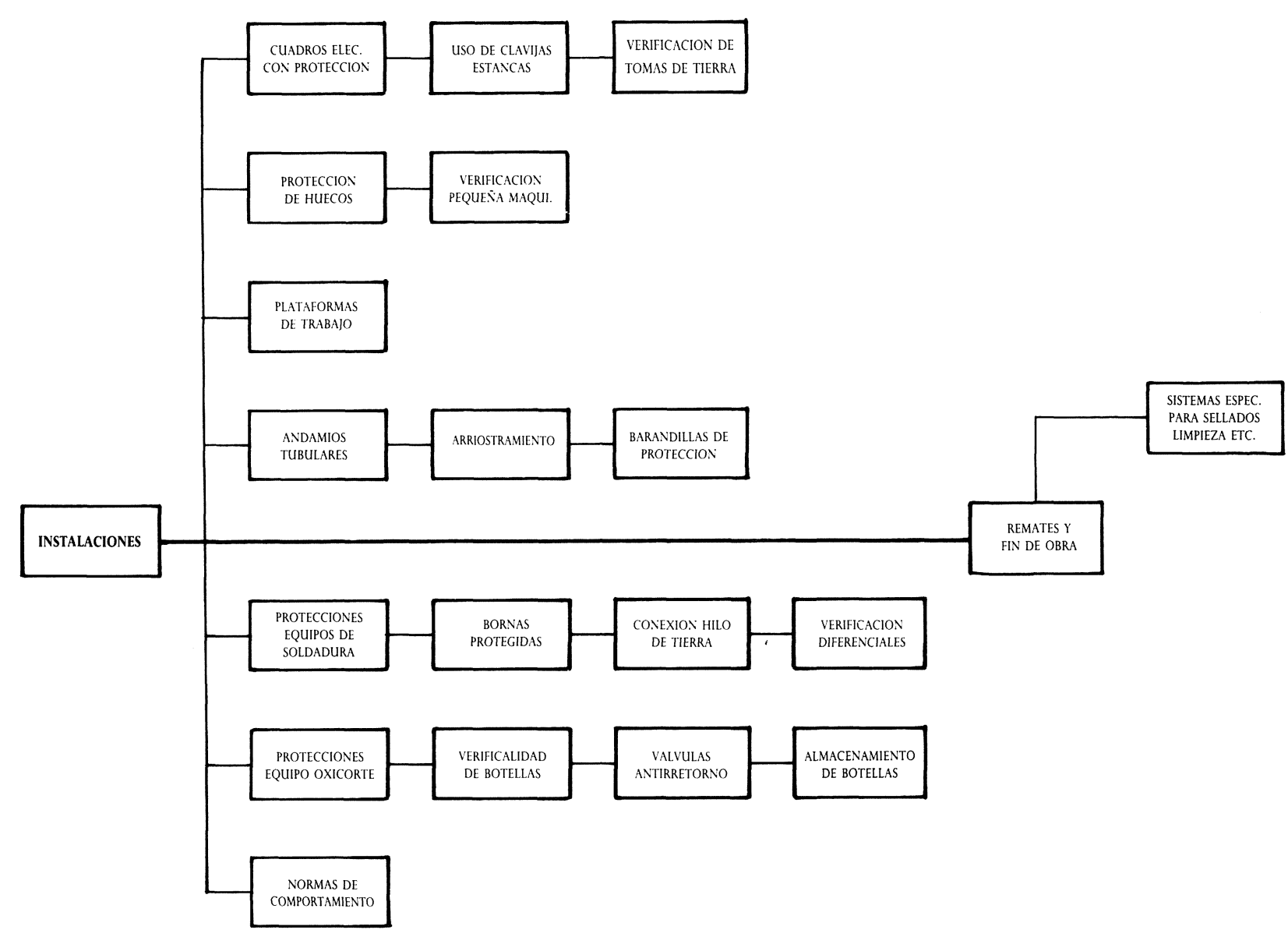

Esquema $70^{\circ}$

\section{$5^{\circ}$ Cubierta}

En la unidad de cubierta habría que destacar la importancia que tienen las cubiertas de gran pendiente, donde será preciso diseñar unos andamios auxiliares en forma de escalera que se adapten a la pendiente de la cubierta, con plataformas de trabajo de $0,60 \mathrm{~m}$ como mínimo, dotadas de barandillas rígidas que eviten caídas al vacío. Este sistema modulado puede ser movido perfectamente por la grúa hasta completar todo el conjunto de la cubierta.

\section{$6^{\circ}$ Distribución interior $\mathrm{y}$ acabados}

En la distribución interior y acabados cabe mencionar, como una de las cosas más significativas, la necesidad del uso de plataformas metálicas de descarga, para evitar, en primer lugar, el roce del cable de la grúa en los extremos de los forjados superiores y, en segundo lugar, para la protección integral del trabajador que tiene que recibir la carga, la cual, a través del traspalé, será movida sin ningún tipo de riesgo hasta el lugar de uso del material elevado. El orden y limpieza en esta unidad de obra son necesarios y fundamentales para evitar el gran número de accidentes que se producen cuando no se cumple esta simple regla de prevención. La evacuación de escombros está ligada a lo indicado anteriormente, y es preciso llevarlo a la práctica a través de las trompas de elefante que para este fin existen en el mercado. Para evitar el ruido, se dispone en el mercado de unos troncos de cilindro en fibra de vidrio que sin ninguna duda bajan el nivel de decibelios. Las protecciones de los huecos existentes tienen que estar constantemente vigiladas por el equipo de seguridad descrito anteriormente. La pequeña maquinaria eléctrica ha de ser siempre empleada con protección de doble aislamiento.

\section{$7^{\circ}$ Instalaciones}

La unidad de obra denominada Instalaciones abarca una serie de elementos industriales, las instalaciones de as- 
censores, eléctricas, aire acondicionado, fontanería, etc...., las cuales tienen factores comunes a lo dicho anteriormente; también por cuanto se refiere a andamios, plataformas, equipos de oxicorte y grupos eléctricos entre los más significativos.

\section{$8^{\circ}$ Remates}

Como cierre a este Esquema Organizativo de edificación, nos encontramos con la última unidad denominada Remates, donde será preciso la utilización de medios especiales para sellados, limpiezas, etc...

\section{CONCLUSIONES}

En resumen, las conclusiones a las que conduce esta ponencia sobre el Proceso Organizativo de la Seguridad son, entre otras:

- La integración de la Seguridad en la Planificación general de la obra.

- La utilización de los medios materiales y mecánicos definidos en el Plan de Seguridad.

- El respeto a las partidas económicas del Plan.

- La consideración del Plan de Seguridad como una herramienta de trabajo en la Prevención.

\section{publicaciones del ICCET/CSIC}

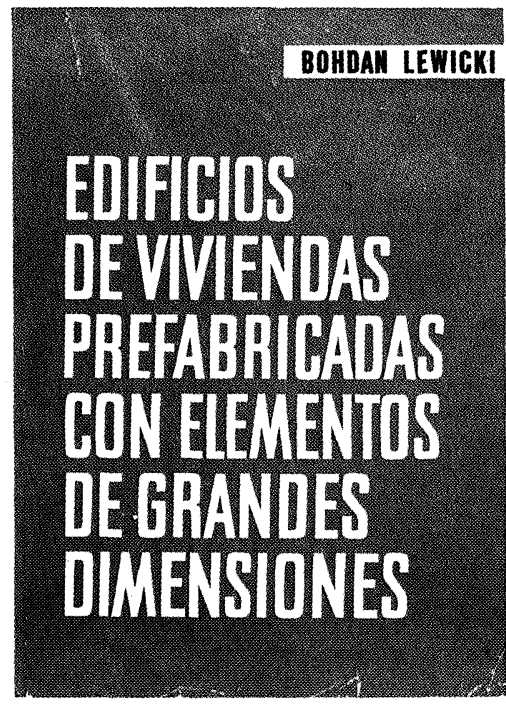

\section{Bohdan Lewicki}

Este libro trata de los problemas relativos a la construcción de los edificios de viviendas o publicos realizados con elementos prefabricados de grandes dimensiones. Se han estudiado los problemas de arriostramiento, asi como los que plantea la resistencia de los elementos y de la estructura; se han examinado las cuestiones de orden higrotérmico, acústico y de resistencia al fuego; también se ha profundizado en el estudio de la estanquidad de los muros exteriores y de las juntas.

La obra incluye numerosas ilustraciones que dan detalles de diversas soluciones, asi como ejemplos de cálculo, tablas de valores numéricos, diagramas y ábacos.

Un volumen encuadernado en tela, de $24 \times 17 \mathrm{~cm}$, compuesto de 616 págs.
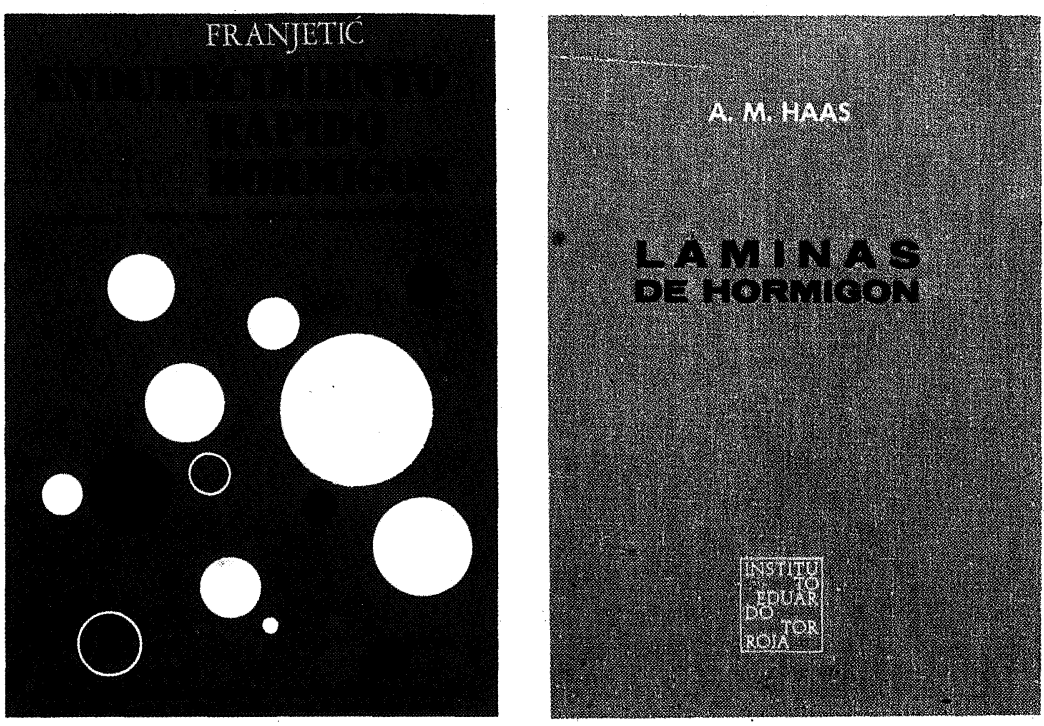

Zorislav Franjetić

En la obra de Franjetić se expone de una forma minuciosa, ordenada y sistemática, todo un cuerpo de doctrina que reúne el conocimiento actual sobre el endurecimiento rápido del hormigón. Parte el autor de los principios básicos y llega a las últimas consecuencias y realidades técnicas y econó micas.

Es una obra de consulta, tanto para el in vestigador sobre la materia, como para el proyectista y el realizador y montador de plantas e instalaciones y equipos de curado y endurecimiento rápido.

Un volumen encuadernado en cartóné, de $17 \times 24,5 \mathrm{~cm}$, compuesto de 385 págs. 110 figuras y 10 tablas.

\section{A. M. Haas}

Al escribir este libro el autor intento pone a disposición de los estudiantes y de los ingenieros unos conocimientos prácticos, adecuados para servir de guía en el diseño y construcción de láminas delgadas de hormigón.

El autor está convencido de que el éxito en el diseño de una lámina exige, por parte del las que pasa la materialización de la lámina: el diseño, el análisis estructural y la construcción de la estructura.

Un volumen encuadernado en tela, de $17 \times 24,5 \mathrm{~cm}$, compuesto de 420 págs., 141 figuras, 22 fotografias y 6 tablas. proyectista, un examen de las tres fases por 